\title{
El Recurso Agua en las Comunidades Indígenas Wayuu de La Guajira Colombiana. Parte 1: Una Mirada desde los Saberes y Prácticas Ancestrales
}

\author{
Alcides R. Daza-Daza ${ }^{(1)^{*}}$, Nelson Rodríguez-Valencia ${ }^{(2)}$ y Alexis Carabalí-Angola ${ }^{(1)}$ \\ (1) Facultad de Ingeniería, Grupo Territorios Semiáridos del Caribe, Univ. de La Guajira, km 5 vía Maicao, \\ Riohacha La Guajira-Colombia (e-mail: adaza@uniguajira.edu.co; acarabali@uniguajira.edu.co) \\ (2) Centro Nacional de Investigación de Café, Cenicafé, Km 4 vía Antigua Manizales, Chinchiná-Caldas- \\ Colombia (e-mail: nelson.rodriguez@cafedecolombia.com)
}

*Autor a quien debe ser dirigida la correspondencia

Recibido Feb. 12, 2018; Aceptado Abr. 19, 2018; Versión final Jun. 21, 2018, Publicado Dic. 2018

\begin{abstract}
Resumen
La finalidad de la investigación fue estudiar los saberes, creencias y prácticas ancestrales que tienen las comunidades indígenas Wayuu de La Guajira Colombiana sobre el tema del agua y como estas manejan y gestionan el recurso para su sostenibilidad. El trabajo se desarrolló dentro del proyecto cofinanciado por Colciencias lineamientos para el ordenamiento territorial costero y marino del departamento de La Guajira. El tipo de investigación es histórica, documental y explicativa. Se utilizó la entrevista como instrumento para colectar la información, la que está compuesta por preguntas abiertas y semiestructuradas. Las preguntas se agruparon en componentes con el fin de facilitar su análisis descriptivo. Los resultados evidencian que las prácticas ancestrales de manejo del agua están supeditadas a la presencia o ausencia del líquido. Esto es que en las zonas con mayor disponibilidad de agua las comunidades tienen comportamientos y costumbres diferentes en términos de administración, conservación y buen uso del recurso, comparado con zonas de escasez de agua.
\end{abstract}

\section{The Water Resource in the Wayuu Indigenous Communities of La Guajira Colombiana. Part 1: A Look from the Ancestral Knowledge and Practices}

\begin{abstract}
The purpose of the research was to study the knowledge, beliefs and ancestral practices that the indigenous Wayuu communities of La Guajira Colombiana have around the concept of water and how they manage this resource for its sustainability. The work was developed within the project co-financed by Colciencias guidelines for the coastal and marine territorial ordering of the department of La Guajira. The type of research is historical, documentary and explanatory. The interview was used as the instrument for gathering information, which includes open and semi-structured questions. The questions were grouped into components to facilitate their descriptive analysis. The results show that the ancestral practices of water management depend upon the presence or absence of the liquid. That is, the areas with better water availability communities have different behavior and customs in terms of administration, conservation and good use of the resource, than those in which water is scarce.
\end{abstract}

Keywords: sustainable management; water management; water resources; ancestral practices; wayuu communities 


\section{INTRODUCCIÓN}

Las comunidades indígenas han adquirido un conocimiento sobre su territorio, producto de la interacción que estos han establecido con el medio ambiente a lo largo de la historia (Opare, 2016a). En este sentido, el conocimiento indígena hace referencia a los saberes y prácticas ancestrales transmitidos por generaciones por medio de la oralidad (Williams y Hardison, 2013; Tomaselli, 2014). Por lo anterior, las comunidades indígenas presentan un gran interés por la gestión de sus recursos hídricos, producto de la relación que existe entre los recursos naturales y los saberes ancestrales, en la que el agua se valora y se maneja según las costumbres aborígenes (Jackson, 2005). Bajo estos principios, los grupos indígenas de las zonas áridas y semiáridas con acceso limitado al recurso agua, han usado su conocimiento y sabiduría humana para el desarrollo de sistemas de gestión integrales dentro de sus territorios (Tahmasebi, 2009). Lo anterior, les ha permitido conservar sus recursos mediante sistemas de manejo tradicional; logrando la sostenibilidad del medio ambiente que estos poseen (Reyes y Martí, 2007). Partiendo de lo anterior, las culturas indígenas fundamentan la gestión de los sistemas naturales en el conocimiento del hábitat natural, por medio de signos e interpretaciones (Karlin, 2016).

Para las comunidades indígenas, el respeto por la naturaleza es la base fundamental en la conservación de los recursos naturales (Camacho et al., 2016). El conocimiento desarrollado a partir de las prácticas ancestrales, contribuye de cierta forma a la restauración de los ecosistemas (Uprety et al., 2012). En este sentido, los sistemas tradicionales brindan información útil para entender las dinámicas socioambientales presentes en los territorios, aportando estrategias de manejo sobre los ecosistemas (Wateau, 2011). Desde este punto de vista, el conocimiento ancestral de los recursos naturales por parte de las comunidades indígenas, les ha permitido conservar sus recursos de forma sostenible a lo largo de la historia, garantizando de esta forma el sostenimiento de sus generaciones (Negi et al., 2017).

El agua para los pueblos indígenas representa un recurso sagrado, compuesto de rituales y ceremonias que armonizan el cosmos y perpetúan las tradiciones. Desde la cosmovisión indígena el agua está íntimamente relacionada con el existir del ser humano. El vínculo hombre, agua y naturaleza hacen parte de la identidad cultural de las comunidades; la presencia o escasez de este recurso influye en las prácticas de manejo que estos implementen (Jiménez et al., 2014). En este sentido, el agua para el Wayuu es un ser vivo que se enmarca muchas veces dentro del simbolismo de los sueños, para revelar hechos relacionales con el medio natural con el que interactúan (Instituto Colombiano de Cultura Hispánica, Geografía Humana de Colombia, 2014). El ser Wayuu está fundamentado en la relación armónica con el mundo natural y el valor sagrado de la vida.

Conviene subrayar, que uno de los mayores problemas actuales que viven los territorios indígenas es la forma como las concepciones locales, simbólicas, cosmológicas y socioculturales chocan con las políticas económicas y técnicas de las instituciones nacionales que plantean los sistemas de gestión desde sus realidades sin tener presente las particularidades de cada territorio (Wateau, 2011). En este sentido, las prácticas culturales pueden ser consideradas opciones viables para enfrentar los problemas de sequía en los territorios; sin embargo, para tener una verdadera incidencia como alternativas de solución es necesario que estas se integren a las políticas económicas y sociales de cada región (Sampaio, 2009). Bajo este precepto, el presente artículo se centró en estudiar los saberes, creencias y prácticas ancestrales de las comunidades indígenas Wayuu de La Guajira Colombiana como una posible herramienta en la gestión sostenible del agua.

\section{MATERIALES Y MÉTODOS}

La metodología se describe en dos subsecciones; en la primera se contextualiza la localización del área de estudio y en el segundo segmento se plantean las herramientas y procedimiento ejecutados para el logro de los objetivos.

\section{Área de estudio}

La zona objeto de estudio se localiza en el extremo norte de Colombia, limitando al norte con el mar Caribe, al sur con el departamento del Cesar, al este con el mar Caribe y Venezuela y al oeste con el mar Caribe y el departamento del Magdalena (Figura 1). El departamento cuenta con 15 municipios y 44 corregimientos. Dentro de las fuentes de agua más importantes se encuentran los pozos, jagüeyes, represas y ríos como el Ranchería, Cesar, Jerez, Ancho y Palomino (Gobernación de La Guajira, 2011). En cuanto a la diversidad étnica y cultural del país; cuenta con un porcentaje aproximado de población indígena que oscila entre el 22 $\%$ al $61 \%$, distribuidos en los 11 municipios con resguardos indígenas (Riohacha, Uribia, Maicao, Dibulla, Distracción, Hatonuevo, Manaure, San Juan del Cesar, Albania y barrancas). De este porcentaje, el 38 \% de la población del departamento son Wayuu (Cámara de Comercio de La Guajira, 2015). 


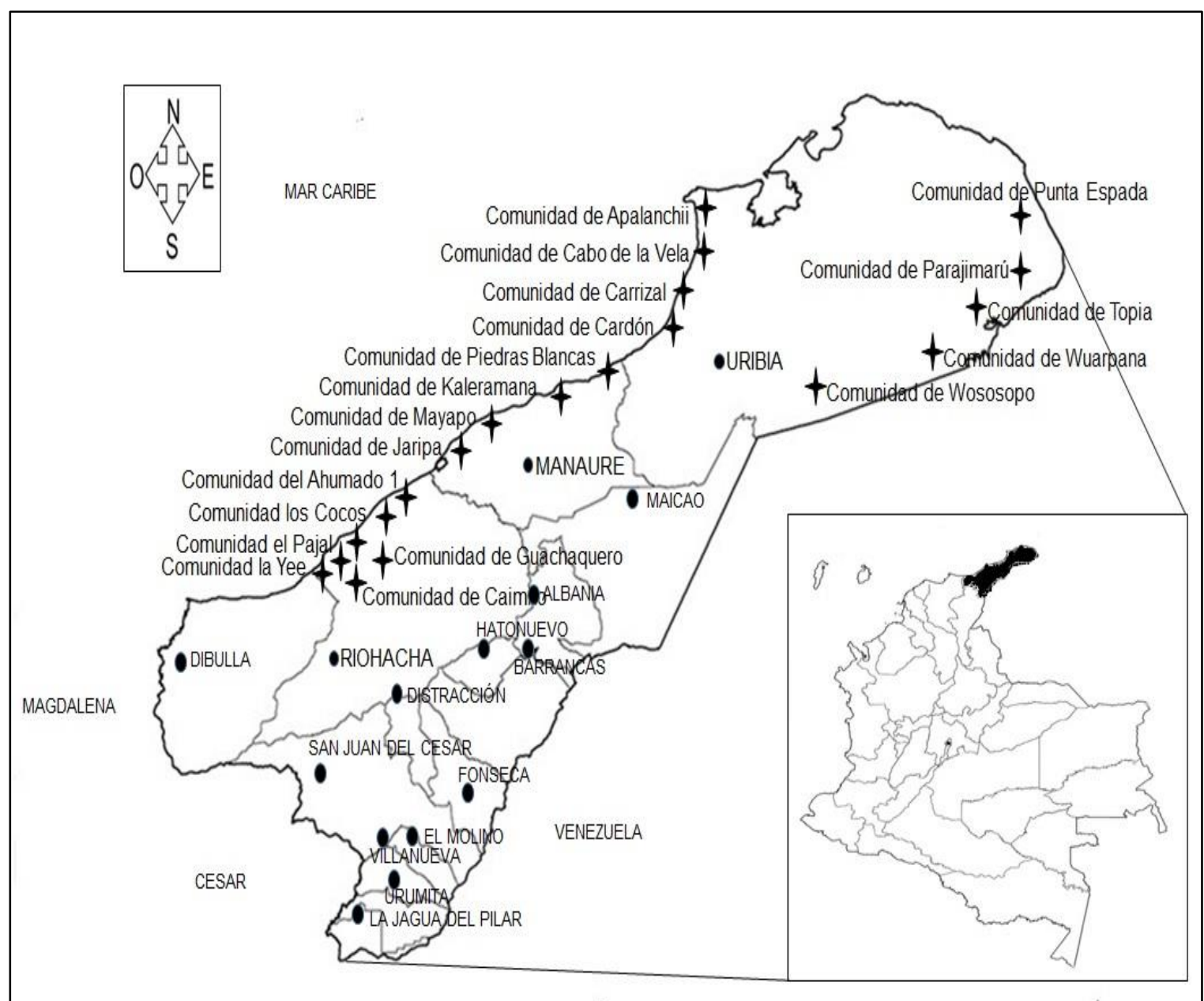

Fig. 1: Localización del área de estudio (Adaptada del Departamento Nacional de Estadística [DANE], 2012)

\section{Aspectos metodológicos}

En el estudio se aplicó la investigación histórica, documental y explicativa; con el fin de entender las costumbres, categorías y tradiciones existentes por parte de las comunidades indígenas Wayuu con respecto al agua. Para esto, primero se realizaron consultas en el Sistema Nacional de Información Cultural de Colombia, el Catálogo de la biblioteca Luis Ángel Arango incluyendo su red de bibliotecas y el archivo documental de la biblioteca Nellith Abuchaibe de la universidad de La Guajira). Igualmente, se efectuaron consultas de artículos científicos relacionados con la cosmovisión, los saberes y las prácticas ancestrales de las comunidades indígenas alrededor del uso, manejo y gestión sostenible del agua. Las bases de datos consultadas fueron Scopus, Scielo y Redalyc. Se visitaron en total 20 comunidades indígenas Wayuu de La Guajira Colombiana, distribuidas en los municipios de Uribia, Manaure y Riohacha; tratando de tener una representatividad de todo el territorio (Figura 1).

Se entrevistaron 30 personas en los que estuvieron pescadores, líderes, autoridades tradicionales, artesanos y educadores. En este sentido, participaron en las entrevistas 18 mujeres y 12 hombres quienes fueron entrevistados de manera directa e intencional con edades que oscilaron entre los 28 a 85 años de edad. Teniendo en cuenta que algunos de los informantes hablaban únicamente la lengua nativa se contó con el apoyo de una indígena Wayuu como traductora. La investigación se desarrolló en el periodo comprendido de agosto a diciembre de 2017. Para garantizar la calidad de los resultados las entrevistas realizadas fueron contrastadas con los aspectos de reconocimiento visual realizados en cada una de las zonas. Previo a cada visita, se realizaron acercamientos con las autoridades tradiciones de cada territorio, con el fin de explicar los intereses del estudio y solicitar las respectivas autorizaciones de ingreso. Con el ánimo de garantizar el manejo de la información, se firmaba con cada líder o autoridad tradicional un acuerdo de consentimiento informado. Todas las entrevistas fueron grabadas previa autorización verbal por parte de las personas entrevistadas, garantizando el anonimato de los participantes, para su posterior transcripción y análisis de la información. Igualmente, se respetó las consideraciones establecidas en la Resolución 8430 del 4 de octubre de 1993 del Ministerio de Salud de Colombia con relación a los tipos de riesgos que están expuestos los sujetos de investigación. Los datos recolectados de las entrevistas fueron codificados, depurados y analizados utilizando el software para análisis cualitativos de datos Nvivo versión 11. 


\section{RESULTADOS Y DISCUSIÓN}

Los resultados se analizaron desde tres planteamientos: primero, se realizó una descripción de la población Wayuu, luego se hizo un recorrido desde la cosmovisión Wayuu alrededor del agua y se finalizó con un análisis enfocado a evaluar el manejo y la gestión del recurso dentro del territorio.

\section{Caracterización de las comunidades indígenas Wayuu}

El grupo étnico Wayuu pertenece a la familia lingüística Arawak, proveniente de las zonas selváticas del Amazonas habitando en las fronteras del Orinoco y los ríos de Brasil (Paz, 2016). Constituyen una de las extensas etnias indias de las zonas bajas de América del Sur. Son un pueblo que durante siglos de luchas con los Caribes fueron desplazados y permanecieron en itinerancia en territorio Venezolano y Colombiano, hasta llegar a tierras baldías de la Alta Guajira; región desértica con vegetación xerofítica (Perrin, 1980). En lo que se refiere a las actividades económicas, el territorio ancestral Wayuu en sus inicios de ocupación estuvo marcado por las actividades de caza, pesca y recolección. Durante el periodo colonial mantuvieron relaciones antagónicas con el imperio español y amistoso con los otros poderes Europeos (Francia, Inglaterra, Holanda entre otros). En este periodo y con la adopción de ganado doméstico basan su economía en la venta y comercialización de caballos, asnos y perlas. La pesca, la caza y la siembra estacionaria aseguraron para la época la subsistencia de la comunidad para finales del periodo colonial y principios de la época republicana. Durante el siglo XX, la población indígena comenzó a sufrir los primeros impactos de la cultura de occidente sobre sus creencias, costumbres y modos de vida.

La ocupación del territorio por los proyectos mineros, la ganadería y la agricultura intensiva, el deterioro de recursos naturales y el conflicto armado lograron dispersar y deteriorar la estructura clanil, ocasionando el desplazamiento de estos a zonas con mejores condiciones de vida. Igualmente, el estado ha declarado algunas áreas de resguardos indígenas como zonas baldías, cediendo títulos a personas ajenas a la cultura Wayuu, relegando aún más las costumbres ancestrales (Instituto Colombiano de Cultura Hispánica, Geografía Humana de Colombia, 2014). Los Wayuu generalmente viven en rancherías (pequeños caseríos) ocupadas por viviendas hechas de barro, cubiertas con techo de Yotojoro (especie de cactus propia de las zonas desérticas, conocido con el nombre científico de Stenocereus Griseus). Actualmente la cultura occidental ha impactado la estructura bioclimática de la vivienda Wayuu, cambiando las condiciones ancestral del techo por láminas de zinc. Internamente las viviendas son pequeñas, distribuidas en dos cuartos, usados con el fin de colgar hamacas, mochilas y demás objetos de uso diario. La cocina Wayuu esta por fuera de la vivienda y son construidas con material local (techos de ramas), cercadas de cactus o madera. Distante de los hogares se encuentran los corrales formados por cercas de cactus y trupíllo para resguardar el ganado ovino y caprino. Cerca de la casa principal se localiza una enramada con postes y techos de Yotojoro denominada (Luma), utilizada en los encuentros familiares y atención de visitantes. Los nombres de las rancherías son dados de acuerdo con el nombre de algún animal, planta o sitio geográfico (Perrin, 1980). Figura 2.

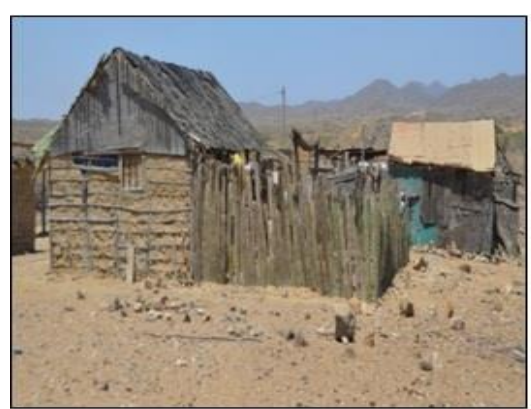

a)

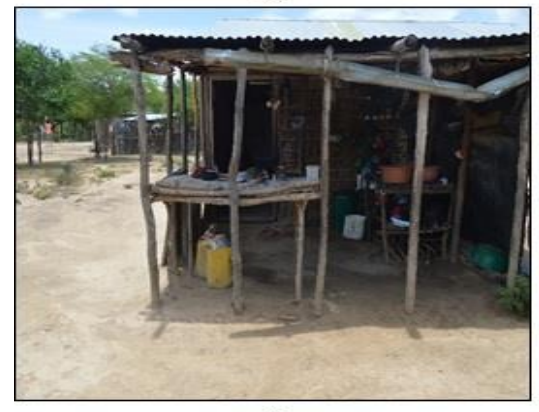

d)

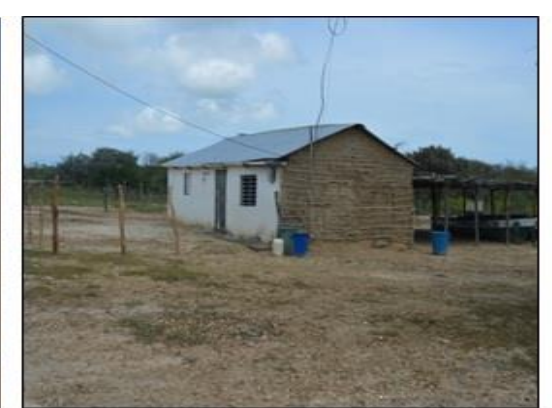

b)

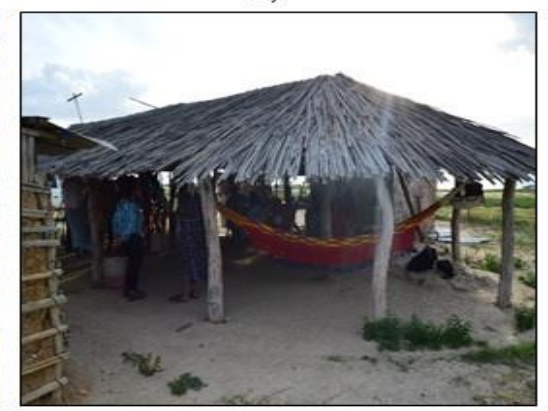

e)

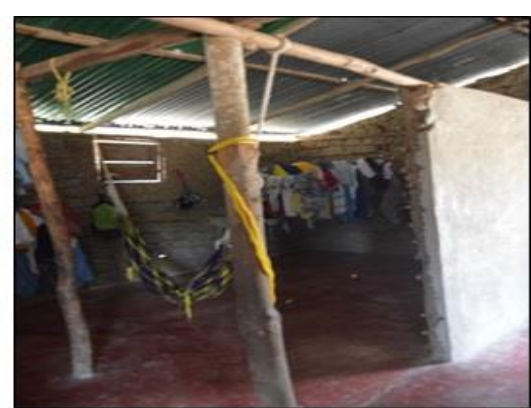

c)

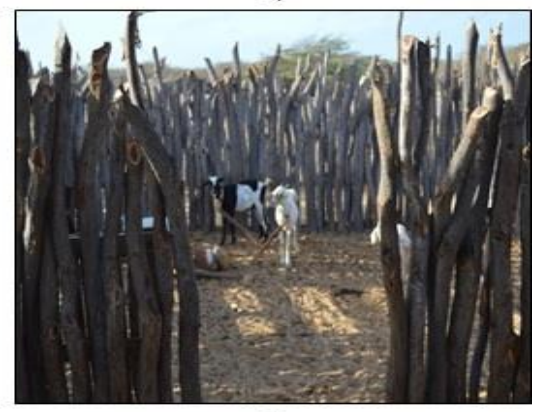

f)

Fig. 2: Características de la vivienda Wayuu: a) Vivienda ancestral; b) Vivienda modificadas; c) Interior de una vivienda; d) Cocina; e) Enramada; f) Corrales 
Por otra parte, la población Wayuu está compuesta clanes matrilineales no exogámicos, presentándose alrededor de treinta clanes cuyos nombres están asociados a animales epónimos (animales quedan nombre a los clanes Wayuu). Existen las personas encargadas de llevar la palabra (buscar conciliación) entre grupos familiares en conflicto. El tío materno es la autoridad tradicional y quien coordina el grupo para la atención de los problemas de la familia. Los hijos son dirigidos por el hermano de la madre y no por su padre biológico. La mujer es la encargada de mantener la cultura tradicional y la interacción de los clanes por medio del matrimonio. De igual modo, la mujer desempeña un papel preponderante dentro del mundo de la espiritualidad de la etnia Wayuu, es la delegada del cuidado de la familia en el hogar, mantiene la armonía, fabrica cerámicas, teje vestidos y mochilas, interpreta los sueños, oficia rituales y es conocedora del poder curativo de las plantas tradicionales (Perrin, 1980). Figura 3.

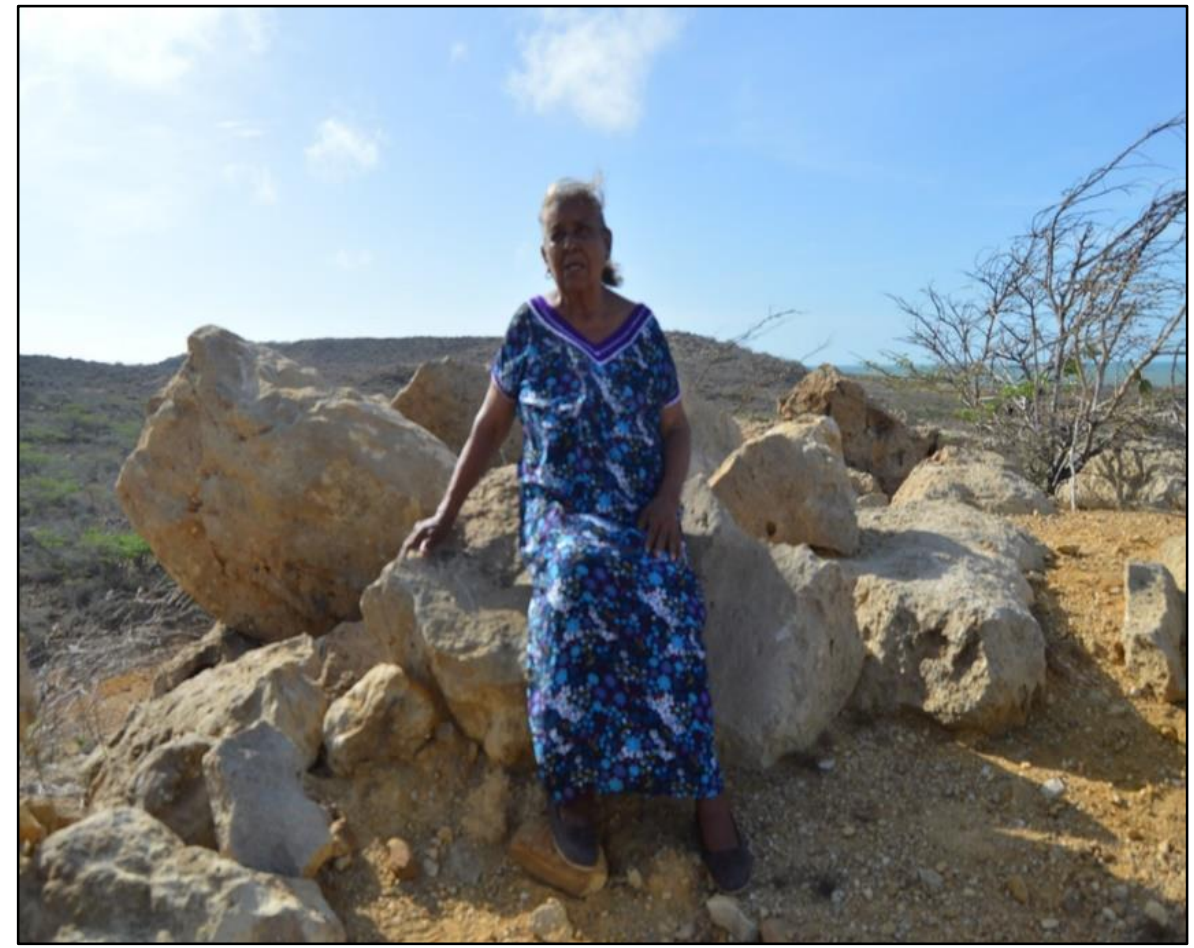

Fig. 3: Mujer Wayuu

La vida en cada territorio está basada en las costumbres, tradiciones y creencias propias de sus realidades sociales, económicas y ambientales presentes en cada espacio geográfico donde habitan. No existe un solo jefe que los gobierne. Los llamados jefes de clanes son personas que dentro de la cultura representan a grupos familiares que por sus condiciones económicas, desempeño, edad, conducta y experiencia toman este rol de autoridad tradicional dentro de la sociedad Wayuu. Cada clan en el departamento de La Guajira tiene su lugar de origen (nacimiento de sus antepasados); igualmente, un clan puede presentar diferentes lugares de asiento (territorios donde decide vivir cada familia). La escogencia del lugar de vivienda es conservada a través de la linea materna; donde su sistema de parentesco sigue la linea de la madre, presidido por un criterio de asentamiento matrilocal; es decir, las parejas construyen sus viviendas cerca de la vivienda de la madre de la esposa (Perrin, 1980). Igualmente, es de aclarar que los Wayuu tienen la costumbre en los periodos de verano extremo de trasladarse a los territorios habitados por familiares uterinos, catalogándolos también como poliresidenciales (Instituto Colombiano de Cultura Hispánica, Geografía Humana de Colombia, 2014). Los Wayuu se rigen a partir de tres clases de parentesco; por consanguinidad cuando están ligados por vínculo de sangre, por afinidad que se presentan por medio de las relaciones matrimoniales y el parentesco clanil, caracterizado por las relaciones entre clanes (Paz, 2016).

De esta forma, el sistema normativo Wayuu utiliza la palabra como elemento fundamental para la resolución de conflictos basado en la compensación (pago de las faltas cometidas). En estos términos, la palabra representa la dignidad y honra de un núcleo familiar o clan (Figueroa y Medina, 2014). Este acto desde el enfoque cultural tiene sus raíces en los lazos establecidos con el medio natural; es decir, el hecho de realizar un pago por alguna falta, que tiene como propósito recomponer las relaciones superando el efecto nocivo de la falta (ofensa). Por otra parte, la lluvia representa un papel importante como elemento dador de vida, es el ser que fertiliza a la tierra y engendra las plantas; las estrellas y la luna son considerados sus hermanos. Así mismo, el sueño dentro de la existencia del ser Wayuu, influye a lo largo de toda su existencia, sirviendo como canal de comunicación con sus ancestros para proteger al grupo familiar mediante mensajes de prevención. 


\section{Cosmovisión de la etnia Wayuu alrededor del agua}

En torno al agua las comunidades indígenas de La Guajira Colombiana establecen un sinnúmero de creencias, saberes y prácticas ancestrales que demarcan la importancia del recurso dentro de la cultura. Parte de esta connotación mitológica milenaria se justifica en la población indígena producto de su escasez, que genera todo tipo de sentimientos de gratitud y respecto ante los incesantes meses de sequía que deben afrontar a lo largo de un año. En este sentido, el agua adquiere un simbolismo y un uso desde la visión Wayuu que a lo largo de esta sección se explicará. Para la mujer (jiet) y el hombre (tooro) Wayuu el agua de lluvia adquiere diferentes significados dependiendo de la época en que se presenta, su frecuencia, la intensidad y los fenómenos meteorológicos que esta pueda traer. La palabra Juya es utilizada con el fin de especificar el agua líquida que traen las nubes, las estaciones lluviosas y los personajes mitológicos de sexo masculino; interpretados, como fecundadores de la tierra. Igualmente, es empleada para denotar los lugares donde se desarrollan los festejos de su llegada y los espacios utilizados para resguardarse de la lluvia. Ahora bien, si en verano se presentan lluvias al momento de dar sepultura aún Wayuu, se considera que el difunto fue agradecido con los designios de juya en vida (Perrin, 1980; Paz, 2016).

Por otra parte, el agua de lluvia (saiña juya) es vista desde dos dimensiones, lo mítigo y lo natural; es decir, el Wayuu desde su connotación del saber empírico y al no tener una respuesta científica de cómo se presenta el fenómeno, lo explica a partir de la interacción y la convivencia de muchos años con el entorno natural (Paz, 2016). Con relación a esto, el ser Wayuu interpreta que Juya (lluvia) menos precia las condiciones orográficas de muchos sectores del departamento de La Guajira (montañas de poca elevación, vegetación escasa, dispersa y de poco tamaño y cobertura, vientos fuertes y suelos áridos) donde los vientos no dejan que el vapor de agua se condense y estas se desplazan a zonas más altas para que Juya fecunde los terrenos y crezcan las semillas. La lluvia es vista como el ser creador de los ríos, lagunas, arroyos, pozos, casimbas, manantiales y corrientes superficiales de agua que permiten alimentar los jagüeyes (Paz, 2016). Lo anterior, deja claro que Juya dentro de la mitología Wayuu es el ser dador de vida, es el sostén y el alivio del pueblo ante los incesantes y agrestes meses de escasez del recurso. Se considera como el padre que permite el renacer de los muertos. En este sentido, en el mundo son muchas las culturas indígenas que han resaltado la importancia mitológica del agua; dando la connotación de ser vivo, de fuente de vida y sangre de la madre tierra, ser integrador donde sus formas de presentación en la naturaleza (lluvia, ríos, agua subterránea, mares) constituyen un solo individuo (Finn y Jackson, 2011). Contrario a esto, la cultura de occidente valora el agua como un recurso económico, sin vida y sin ningún tipo de significado espiritual (Nash, 2007).

\section{El saber Wayuu y los indicadores naturales en torno al agua}

Es necesario, aclarar que a pesar que la lluvia (Juya) es representada por el Wayuu con un ser de vida, también existen momentos de la cotidianidad donde Juya es concebida como un ser que emana temor. Esto significa, que existen ciertos acontecimientos derivados de la lluvia que generan este tipo de comportamientos en la cultura. Como ejemplo se pueden citar los presagios relacionados con las tormentas; en este sentido, el Wayuu coloca al frente de su vivienda machetes en forma de cruz con el fin de calmar el mal tiempo. De igual forma, los ancianos lanzan al aire injurias contra las tempestades en sentido de regaño por su mal comportamiento. Otro aspecto de resaltar, es la creencia de agitar una manta larga para intimidar a las nubes que ocasionan tempestades. De acuerdo con lo anterior, es claro evidenciar que los periodos de mucha pluviosidad son asimilados por la etnia como el principio de enfermedades y muerte. La presencia de mucha agua genera la proliferación de insectos, problemas respiratorios e infecciones en la piel (Paz, 2016).

Hay que mencionar además, que para el Wayuu la terminación de la época de verano es un acontecimiento de alegría, que genera tributo por parte de estos porque es el inicio de la llegada de las primeras lluvias, lo que hace que estos ofrezcan sus primeras cosechas a Juya; realizando bailes (Yonnas) y comidas en su nombre. Es de resaltar también, que en los meses en el que el Wayuu acostumbra a esperar las lluvias y estas se retrasan, proceden a realizar de igual forma bailes implorando a Juya su pronta llegada. De igual forma, ciertas comunidades indígenas Wayuu de la Alta Guajira implementan ritos para evocar la lluvia. Este rito consiste en cubrir los orificios hechos por los iguánidos (cierto tipo de reptiles caracterizados por presentar escamas, de cabeza grande y cola larga) en los arboles con barro para impedir su salida y evitar que perturben a juya. Previo a esto, la comunidad se reúne y ejecuta una gran fiesta para conmemorar la realización del ritual (Perrin, 1980). Otro rasgo para señalar se relaciona con la capacidad que tiene el Wayuu de interpretar su entorno natural para pronosticar la llegada de la estación húmeda. En este sentido, el canto incesante de las guacharacas (especie de ave galliforme de la familia Cracidae), el desplazamiento de las hormigas, el apareamiento masivo de mamíferos (chivos) y la generación de forma periódica de remolinos de arena a lo largo de los días son etnoindicadores ambientales de este fenómeno. De igual forma, existen ciertas creencias relacionadas con las especies de reptiles y las tempestades, se considera que los lugares donde abundan las serpientes son propensos a la presencia masiva de tormentas eléctricas (Paz, 2016). 


\section{Manejo sostenible del agua por parte de las comunidades indígenas Wayuu}

En los aspectos de uso y manejo del agua por parte de las comunidades indígenas, se tiene que algunas de sus actividades giran en torno al trabajo comunitario, donde cada uno de los integrantes del núcleo familiar desempeña un papel específico. En este sentido, la población ha establecido un vínculo con el recurso, siendo este esencial para sus vidas. El análisis que a continuación se presenta se orienta en plasmar una revisión de los antecedentes históricos relacionados con el manejo del agua desde el saber y las prácticas ancestrales. Igualmente, se contempla una interpretación a partir de la relación naturaleza, sociedad, cultura y escasez. En este orden de ideas, el agua es utilizada en muchos rituales y adquiere un manejo especial dependiendo de su uso. En cuanto a esto, se tienen las siguientes connotaciones.

La etnia Wayuu al experimentar la partida de un ser querido desarrollan rituales previo de su entierro. En relación con esto, son las mujeres dentro de la cultura las encargadas de asumir este papel y deben tener algún vínculo familiar al momento de realizar esta labor. Igualmente, las Wayuu deben ser mayores de edad, sin hijos pequeños y no pueden después de cumplido el acto cocinar o tocar a los niños, evitando que queden expuestos a enfermedades. Dentro de la cultura esta tarea es una de las más difíciles por la concepción que tiene el Wayuu que al ejecutar estas prácticas con el recién fallecido quedan propensas a los espíritus. El difunto se higieniza completamente con la finalidad que pase a una mejor vida (Paz, 2016). El agua empleada se mezcla con sal antes de ejecutar el aseo del cuerpo. De igual forma, en la bóveda como creencia le colocan un recipiente con agua evitando que el fallecido pase sed en la otra vida. Las mujeres después del lavado del difunto se duchan en ron o chirrinchi (bebida alcohólica elaborada de forma artesanal por el Wayuu a base de la fermentación de la panela) para liberarse de toda enfermedad y atadura (Chacín, 2016). Antiguamente, el muerto era metido y limpiado en una batea de madera y las aguas de residuos se vertían a las afueras de la vivienda en dirección donde el muerto seria enterrado. Lo dicho hasta este momento, evidencia los cuidados higiénicos que enmarca el Wayuu en la realización de este tipo de prácticas. El lavado de su cuerpo con ron o chirrinchi y el evitar que las mujeres manipulen alimentos permiten evidenciar hábitos higiénicos de manejo. De igual forma, es de resaltar que después del entierro y antes de consumir los alimentos en honor del muerto, los hombres proceden al lavado de sus manos como habito de limpieza.

Otro de los rituales de gran importancia donde el agua adquiere un papel significativo se relaciona con el paso que tiene la Wayuu de niña a mujer adulta. En el desarrollo de la tradición el agua es usada en la preparación de bebidas (Jawapi, Kaswo'u y Palisse) que la limpian de impurezas el cuerpo y la mantiene joven. También son sometidas a baños durante todo el día (tres en el día y uno en la madrugada con la luz de la luna). La labor solo es desarrollada por mujeres (las abuelas y la madre). El agua empleada se almacena en múcuras (recipiente elaborado de barro de forma artesanal para almacenar agua) puestas al sereno con el fin de enfriarla y retirar de su mente los malos pensamientos. Cuando la Wayuu queda lista es sacada en la madruga y la madre o la abuela toman buchadas de agua fría y se la esparcen por todo el cuerpo buscando tonificar su piel y liberarla de malos humores. Las múcuras deben estar sin uso al igual que las totumas (recipiente elaborado en calabazo seco utilizado como utensilio en el hogar Wayuu) que se utilicen para dar los brebajes (Paz, 2016).

Igualmente, en la preparación de los alimentos, las labores agrícolas y la etnomedicina el agua recibe un valor importante dentro de la cultura. En este sentido, la gastronomía Wayuu se caracteriza por la elaboración de sopas (frijol, maíz, millo entre otros), hervidos (lentejas, yuca, pastas de maíz, pasta de millo, guineos entre otros) y bebidas (chicha de maíz y de millo entre otros) que garantizan el sostenimiento de la etnia. De igual modo, algunas comunidades utilizan en periodos de abundancia el agua para el riego de sus cultivos procedente de los jagüeyes, cauces y pozos. (Paz, 2016). Es de aclarar, que en épocas de verano las condiciones cambian. En la construcción de las viviendas el agua también desempeña un papel importante; estas son diseñadas utilizando agua, barro, arcilla, yotojoro (corazón seco del cactus), paja y la técnica de construcción en bahareque (pared de palos entretejidos). De igual forma, existe una característica particular con relación a la ubicación de las viviendas y es que estas se sitúan en zonas altas y retiradas de las fuentes de abastecimiento de agua (Jagüey), producto que el Wayuu tiene la creencia que por las noches los espíritus de Pulowi (ser sobrenatural) deambulan buscando hombres para enamorar mediante artimañas para luego causarles la muerte.

\section{La gestión del agua en las comunidades indígenas Wayuu y su comparación con otras etnias}

Para abordar este punto, es necesario primero conocer cuáles han sido los medios y los sistemas de suministro de agua de las comunidades indígenas. Las dinámicas de ocupación del territorio Guajiro por parte de los Wayuu tuvieron una fuerte influencia del recurso hídrico para su aprovisionamiento. En este sentido, el río Ranchería y los causes que lo alimentan son a lo largo de la historia la despensa de sostenimiento de la población Wayuu. Adicional a esto, el agua de lluvia, las casimbas, lagunas naturales y la utilización del agua almacenada en depresiones naturales de rocas y del suelo fueron otro medio de abastecimiento empleado 
por los aborígenes para su subsistencia. Con el pasar del tiempo y la inclusión de la cultura de occidente aparecieron otras prácticas de abastecimiento de agua como fueron los pozos subterráneos, jagüeyes artificiales, albercas y el empleo de grandes tanques de plástico y carros cisternas que los abastece de agua (Gobernación de La Guajira, 2011).

Por otra parte, antiguamente en las épocas con fuertes tempestades se construían sistemas de goteras (orificios en las viviendas) y de vasijas con tapas (totumas y múcuras) para aprovechar el preciado líquido. De igual forma, muchos Wayuu conservan la costumbre para largos viajes de portar un recipiente elaborado de totumo (lita) donde almacenan agua para beber. Es de aclarar, que dependiendo de su tamaño tiene un uso específico; es decir, aquellos que son de tamaño mediano (diámetro 10 a $15 \mathrm{~cm}$ ) son empleadas para beber agua, leche y bañarse. Las de gran tamaño son utilizadas para trasportar mayores volúmenes de agua. Igualmente, tienen totumas de menor tamaño para tomar café. Desde la costumbre ancestral algunos indígenas utilizan y portan con ellos sus utensilios y recipientes personales de beber y comer para evitar adquirir los males y defectos de las demás personas (Perrin, 1980; Paz, 2016). De lo anterior, se puede decir que existen hábitos de higiene; además, se evidencia como el agua de lluvia es percibida como un recurso de sostenimiento para enfrentar la escasez de los largos periodos de sequía.

En lo que respecta al control, uso y manejo físico del agua son las mujeres, los niños y niñas las encargadas de buscar y acarrear los recipientes como múcuras (utilizado ancestralmente) y tanques plásticos (pimpinas de 20 a 25 litros) con agua. Muchas veces, si no cuentan con animales (asnos) la mujer Wayuu le corresponde realizar largas caminatas para llegar a los pozos, casimbas o jagüeyes (Figura 4). Conviene decir, que existe actualmente una alta influencia de la cultura de occidente para el acarreo de los recipientes en algunas zonas del territorio Wayuu; utilizando motos, carretillas y ciclas. Lo anterior, permite ver el papel preponderante que juega la mujer Wayuu alrededor del agua.

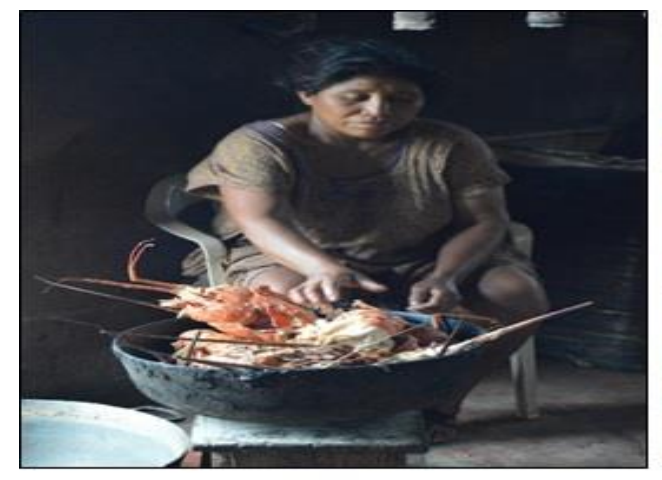

a)

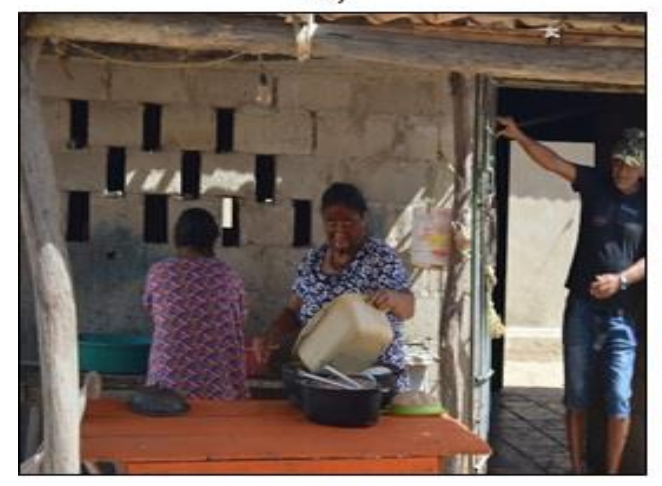

c)

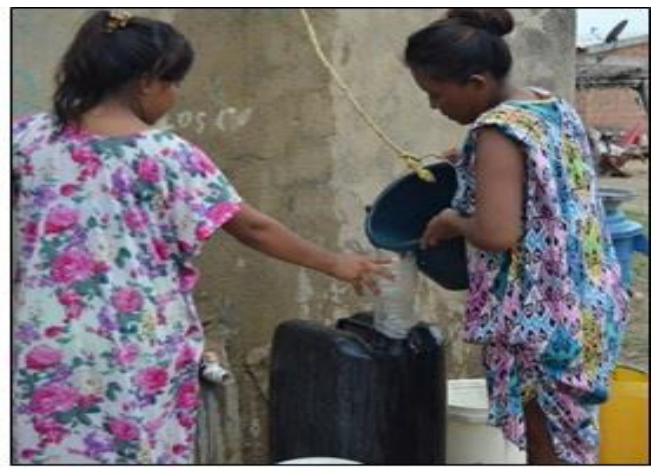

b)

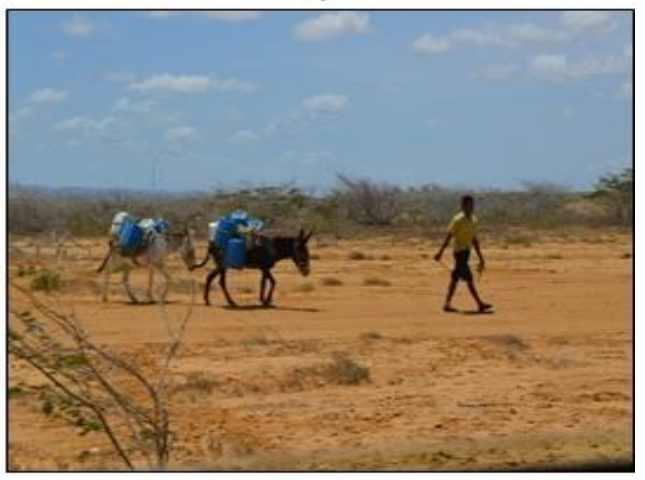

d)

Fig. 4: El rol de las mujeres y niños Wayuu en torno al agua: a) Preparación de alimentos; b) Recolección de agua por las mujeres; c) Lavado de utensilios de cocina; d) Recolección de agua por niños

Habría que decir también, que cuando las condiciones de sequía se vuelven extremas y en los sistemas de abastecimiento el agua escasea o se hace mínima la población emprende estrategias de subsistencia. Es así, que en pocas de verano las familias se desplazan a los jagüeyes secos y construyen casimbas y pozos artesanales alrededor de estos con el fin de extraer del suelo más profundo el agua que se infiltra. Es de resaltar, que en su mayoría estas aguas son turbias, salobres y de mala calidad. En algunas comunidades, muchos han optado por construir sus casimbas y pozos dotados de tapas con cerramientos para que solo los miembros del mismo clan tengan acceso al agua y evitar de igual forma que el agua se contamine. Asimismo, en las entrevistas realizadas se encontró que en época de sequía las comunidades indígenas Wayuu implementan estrategias de control con relación a la cantidad de agua que puede tomar cada familia. En este 
aspecto, es necesario resaltar que en lo referente a las fuentes de abastecimiento de agua, como es el caso de jagüeyes y pozos construidos por los entes gubernamentales presentes en las comunidades indígenas pueden ser usados por miembros de otras comunidades indígenas Wayuu cuando en sus territorios el recurso hídrico escasea. Para lo anterior, las familias de clanes diferentes deben solicitar de manera verbal a la autoridad tradicional el permiso para que sus animales (chivos) y miembros del núcleo familiar puedan acceder al cuerpo de agua para abastecerse. Cuando los miembros ajenos a la comunidad captan el recurso de los jagüeyes y pozos sin previo consentimiento de la autoridad tradicional, el acto es catalogado como una falta que se hace meritoria aun llamado de atención por parte de la autoridad. Si el suceso es repetido se toma como falta grave por parte de la autoridad lo que se hace meritorio al envío de la palabra por medio del palabrero (Putchipü).

En estos términos, el palabrero con los miembros de la comunidad establecen un dialogo estipulando el monto a pagar por la falta cometida, en la mayoría de los casos el pago se realiza en especie (Instituto Colombiano de Cultura Hispánica, Geografía Humana de Colombia, 2014). De igual forma, cuando en el territorio se han presentado proyectos que puedan impactar las condiciones naturales de los recursos hídricos que sirven como fuentes de abastecimiento para las comunidades, como es el caso de la desviación del río Ranchería y los arroyos que lo alimentan producto de las actividades mineras, los Wayuu (Asociación de Cabildos Indígenas Wayuu) junto con los movimientos sociales de carácter local, regional, nacional e internacional han emprendido marchas y acciones legales que han logrado frenar y defender la sostenibilidad del río frente a las iniciativas de explotación minera que imperan en Colombia. Como ejemplo de estrategias para el control de conflictos relacionados con el agua, se puede nombrar las implementadas por las comunidades indígenas de Filipinas, donde el uso de los recursos hídricos y naturales está regido por las prácticas y normas consuetudinarias establecidas por los miembros dentro de su territorio (Rola et al., 2016).

De igual forma, es necesario resaltar que ciertos factores naturales, antrópicos y mitológicos permiten evidenciar un uso eficiente del recurso por parte de las comunidades indígenas Wayuu. Las largas distancias que deben recorrer, el resplandor incesantes del sol, el hecho de que la mujer y los niños cumpla el rol de su acarreo (de una a cuatro pimpinas según sea el medio de acarreo), la carencia de otros medios de carga y el temor a estar cerca de las fuentes de agua durante la noche contribuye a tener una mayor disponibilidad de agua en las fuentes de abastecimiento. Con respecto a esto, Mooney et al. (2012) considera que las culturas indígenas mediante sus creencias establecen vínculos muy fuertes con el agua, lo que garantiza la conservación y sostenibilidad del recurso. Un ejemplo claro de estos aspectos, tiene que ver con el papel que desempeña el sueño dentro de la cultura Wayuu. Cuando los espíritus revelan a través de los sueños los lugares con presencia de agua la comunidad emprende todo un ritual en la búsqueda de la fuente de agua. Generalmente, el lugar se localiza cerca de zonas con presencia de vegetación o lugares por donde fluyen escorrentías superficiales durante las épocas de lluvia. Durante la excavación de la fuente de abastecimiento el Wayuu realiza ofrendas (sacrificio de un chivo ofreciendo su sangre) para pedir permiso a los espíritus.

En este sentido, al igual que en la cultura Wayuu, existen territorios presentes en otras comunidades indígenas donde la espiritualidad juega un papel importante, caso particular se evidencia en el pueblo de Ghana (África) durante la época precolonial, en la que existía la creencia por parte de sus habitantes que los espíritus de sus antepasados eran los encargados de proteger sus recursos hídricos. Las tradiciones mitológicas eran la base para el manejo de los recursos naturales, delegando sobre los líderes espirituales el cuidado de los cuerpos de agua (Agyenim y Gupta, 2013). Acorde con lo anterior, Negi (2017) considera que en muchas culturas no solo africanas, sino también de la India y Asia, la gestión y cuidado de la naturaleza está influenciada por las creencias religiosas de sus habitantes. Vale la pena decir, que para los pueblos indígenas de América del Norte (Anishinaabe) las ceremonias espirituales se realizan para fortalecer los vínculos entre el ser humano, el cosmos y los recursos naturales, en el que el agua representa la vida de todo lo existente (Chief et al., 2016).

Por otra parte, dentro de las estrategias de conservación de las fuentes de abastecimiento de agua como es el caso de los jagüeyes, casimbas y pozos se implementan actualmente sistema de cercos con el uso de alambre y madera (Figura 5). Ancestralmente las comunidades implementaban vegetación xerofítica alrededor de los jagüeyes con el fin de impedir el ingreso de animales. Asimismo, realizan prácticas de limpieza llevadas a cabo por los hombres con el fin de retirar la maleza presente en los reservorios. En este sentido, se puede decir entonces que el conocimiento tradicional en términos de gestión del recurso les ha permitido a las comunidades subsistir a lo largo de la historia dentro de sus territorios. De acuerdo con esto, estudios realizados en la comunidad indígena de Dupong (África Occidental) evidencian como la población en los periodos de sequía implementa estrategias de adaptación para garantizar la disponibilidad del recurso agua en las fuentes de abastecimiento. Para esto, disminuyen el consumo de agua, reutilizan las aguas residuales, los hombres asumen el rol de búsqueda de nuevas fuentes de agua y en conjunto la comunidad desarrolla acciones de mejoramiento de las fuentes de agua existentes (Opare, 2016b). 


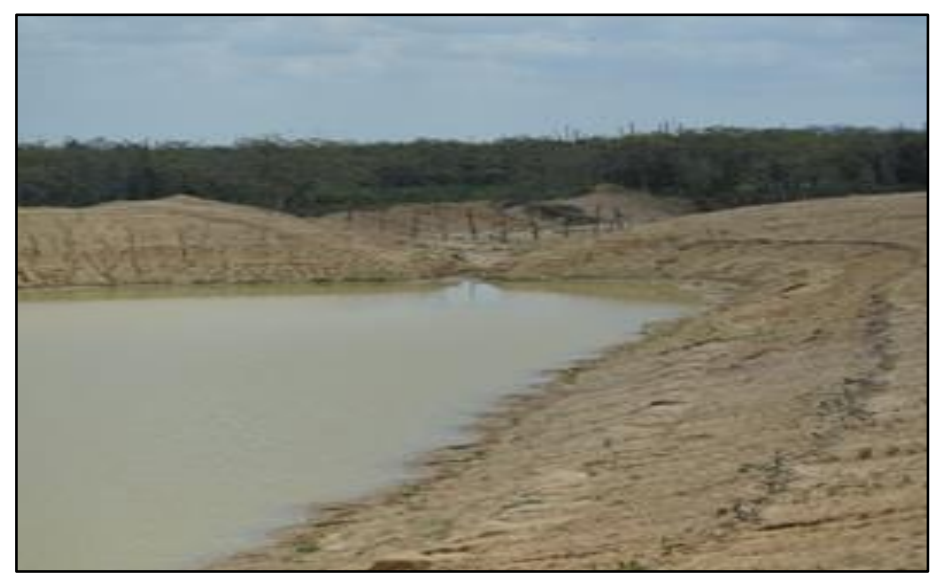

Fig. 5: Estrategias de conservación de jagüeyes con cercas

La apropiación de la población indígena de sus prácticas y saberes les genera pertenencia sobre sus recursos hídricos, contribuyendo esto con su sostenimiento y buen uso del mismo (Behailu et al., 2016). Por su parte, Singh (2006) afirma que el simbolismo que hacen las comunidades indígenas alrededor del agua genera todo tipo de valores, normas y creencias que rigen la vida cotidiana de las poblaciones aborígenes, generando estrategias de gestión del recurso agua desde una visión material e inmaterial.

En cuanto a las prácticas agrícolas realizadas por la población Wayuu, son de tipo estacional contribuyendo al uso sostenible del agua. Las siembras generalmente se desarrollan en los periodos de lluvias, realizando cultivos que ofrezcan productividad en corto tiempo (Figura 6). En lo referente a posibles efectos de contaminación de los cuerpos de agua por el uso de agroquímicos, se tiene que aún se usan prácticas ancestrales para el manejo de plagas, como es el caso de la implementación de la ceniza. Otro aspecto de resaltar, es que el Wayuu no es muy dado al diseño de obras hidráulicas para el riego de los cultivos por las razones antes mencionadas; sin embargo, diseñan de forma artesanal canales con rocas para conducir el agua de escorrentía hacia los jagüeyes. Por lo que se refiere a esto, existen comunidades asentadas en la región de Nepal en las fronteras de India y China donde el uso del agua para las actividades agrícolas se distribuye y maneja según el parentesco y las creencias cosmológicas. En este aspecto, la repartición del agua se fundamenta en una distribución equitativa del recurso según el linaje, donde prima la armonía social, la organización ancestral y el simbolismo entre los miembros que integran el territorio (Wateau, 2011).

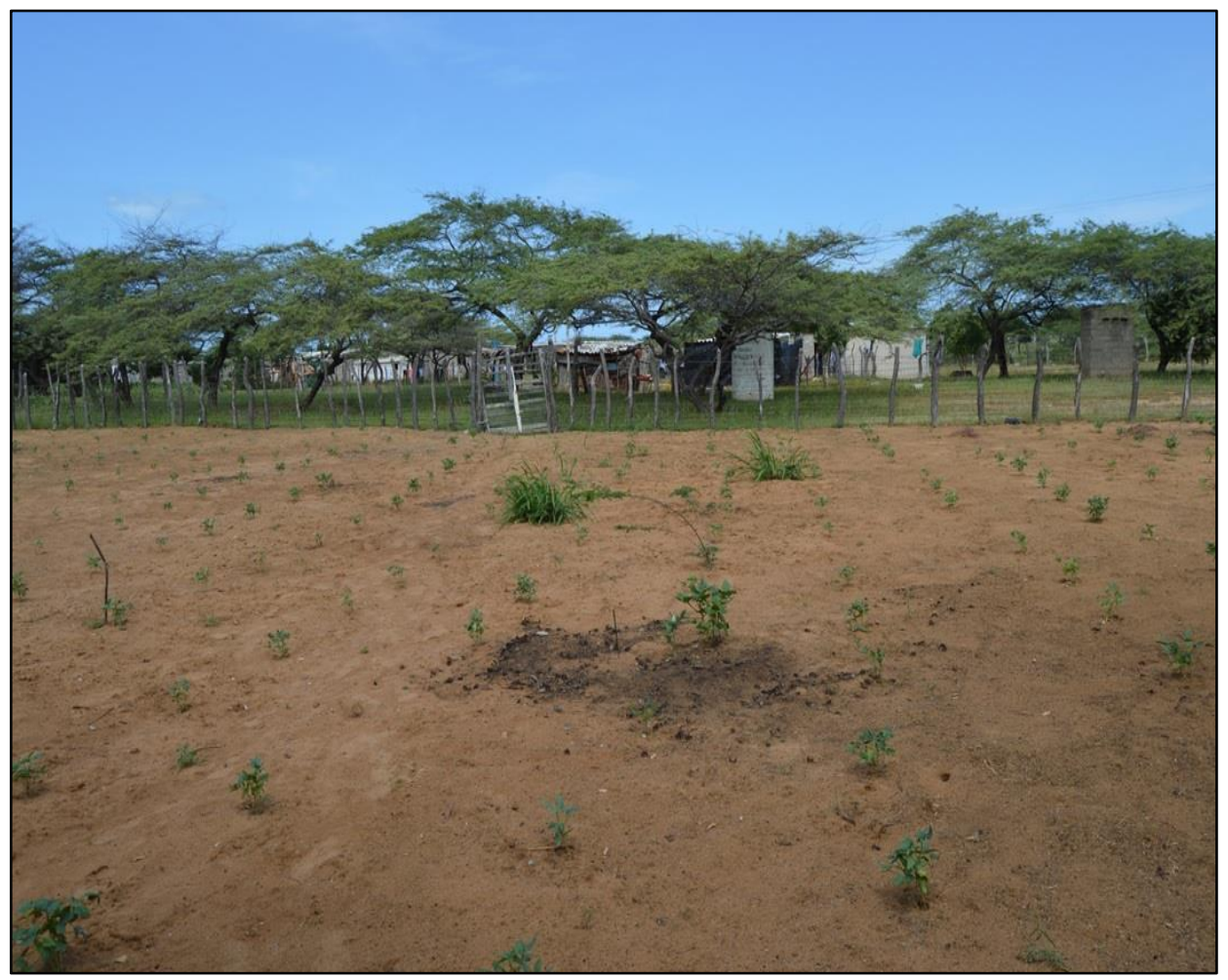

Fig. 6: Huerta 


\section{CONCLUSIONES}

De acuerdo a los resultados y análisis del estudio se logran extraer las siguientes cuatro conclusiones principales:

1) las particularidades y diferencias que puedan existir en el territorio en torno a las prácticas ancestrales de manejo del agua están supeditadas a la presencia o ausencia del líquido; es decir, las zonas con mejores condiciones de disponibilidad de agua optan por comportamientos y costumbres diferentes en términos de administración, conservación y buen uso del recurso;

2) las creencias mitológicas de las comunidades indígenas alrededor del agua garantizan en muchas ocasiones la sostenibilidad de las fuentes de abastecimiento; en este sentido, la población Wayuu valora el agua como un ser generador de vida y bienestar para los territorios;

3) la escasez física del agua se subsana en algunas comunidades indígenas Wayuu gracias a los vínculos parentales, los grupos familiares se desplazan donde los parientes uterinos por provisión de agua y permanecen en ese territorio mientras pasa la crisis en sus lugres de residencia;

4) las acciones de manejo que las comunidades indígenas Wayuu hacen del agua dentro de sus territorios, además de contribuir con su sostenibilidad, permiten el establecimiento y conservación de los vínculos de asociación entre grupos familiares y clanes diferentes.

\section{REFERENCIAS}

Agyenim, J.B. y J. Gupta, Water Management in Ghana: Between the Idea and the Implementation, doi: 10.1080/19390459.2012.668100, Journal of Natural Resources Policy Research, 5(1), 35-48 (2013)

Behailu, B.M., E.P. Pekka y S.K. Tapio, Indigenous Practices of Water Management for Sustainable Services: Case of Borana and Konso, Ethiopia, doi: 10.1177/2158244016682292, Sage Publications, 6(4), 1-11 (2016)

Camacho, L.D., D.T. Gevaña, A.P. Carandang y C. Sofronio, Indigenous knowledge and Practices for the Sustainable Management of Ifugao Forests in, doi: 10.1080/21513732.2015.1124453, International Journal of Biodiversity Science, Ecosystem Services y Management, 12(2), 5-13 (2016)

CDC, Cámara de Comercio de La Guajira, Informe Socioeconómico del Departamento de La Guajira 2015, CDC (2015)

Chacín, H., Asombros del Pueblo Wayuu, 1aㅡ. Ed., 1-150, Fondo Editorial Unermb, Maracaibo, Venezuela (2016)

Chief, K., A. Meadow y K. Whyte, Engaging Southwestern Tribes in Sustainable Water Resources Topics and Management, doi: 10.3390/w8080350, Water, 8(8), 350 (2016)

DANE, Departamento Administrativo Nacional de Estadística, DANE (2012)

Figueroa, N. y O. Medina, Mirada a la Cultura Wayuu, Base de su Sistema Normativo, Revista Verbum, 9(9), 109-117 (2014)

Finn, M. y S. Jackson, Protecting Indigenous Values in Water Management: a Challenge to Conventional Environmental flow Assessments, doi: 10.1007/s10021-011-9476-0, Ecosystems, 14(8), 1232-1248 (2011)

Gobernación de La Guajira, Evaluación Social y Plan de Pueblos Indígenas, para el Proyecto "Construcción y Sostenibilidad de once (11) Reservorios y un (1) Micro Acueducto, en Comunidades Indígenas de la Alta Guajira", Gobernación de La Guajira (2011)

ICCH, Instituto Colombiano de Cultura Hispánica, Geografía Humana de Colombia, Nordeste Indígena, 1르., 215-292, $\mathrm{ICCH}$, Bogotá, Colombia (2014)

Jackson, S., Indigenous Values and Water Resource Management: A Case Study from the Northern Territory, doi: 10.1080/14486563.2005.10648644, Australasian Journal of Environmental Management, 12(3), 136-146 (2005)

Jiménez, A., M. Cortobius y M. Kjellén, Water, Sanitation and Hygiene and Indigenous Peoples: a Review of the Literature, doi: 10.1080/02508060.2014.903453, Water International, 39(3), 277-293 (2014)

Karlin, M.S., Ethnoecology, Ecosemiosis and Integral Ecology in Salinas Grandes (Argentina), Etnobiologia, ISSN: 16652703, 14(1), 23-38 (2016)

Mooney, C. y P.L. Tan, South Australia's River Murray: Social and Cultural Values in Water Planning, doi: 10.1016/j.jhydrol.2012.04.010, Journal of Hydrology, 474, 29-37 (2012)

Nash, J., Consuming Interests: Water, Rum, and Coca-Cola from Ritual Propitiation to Corporate Expropriation in Highland Chiapas, doi: 10.1525/can.2007.22.4.621, Cultural Anthropology, 22(4), 621-639 (2007)

Negi, V.S., R. Pathak y otros cuatro autores, Traditional Knowledge and Biodiversity Conservation: a Case Study from Byans Valley in Kailash Sacred Landscape, India, doi: 10.1080/09640568.2017.1371006, Journal of Environmental Planning and Management, 1-22 (2017) 
Opare, S., Practising the Past in the Present: Using Ghanaian Indigenous Methods for Water Quality Determination in the Contemporary Era, doi: 10.1007/s10668-016-9851-2, Environment, Development and Sustainability, 19(6), 2217-2236 (2016a)

Opare, S., Adaptation to Climate Change Impacts: Coping Strategies of an Indigenous Community in Ghana to Declining Water Supply, doi: 10.1080/17565529.2016.1184610, Climate and Development, 10(1), 73-83 (2016b)

Paz, R., Concepción y Descripción de la Cultura Wayuu, 1aㅡ Ed., 1-362, Fondo Editorial Wayuu Araurayu, Bogotá, Colombia (2016)

Perrin, M., El Camino de los Indios Muertos, Mitos, Símbolos Guajiros, 1를 Ed., 1-272, Monte Ávila Editores, Caracas, Venezuela (1980)

Reyes, V. y N. Martí, Etnoecologia: Punto de Encuentro entre Naturaleza y Cultura, Ecosistemas, ISSN: 1132-6344, 16(3), 46-55 (2007)

Rola, A.C., C.L. Abansini y otros cuatro autores, Drivers of Water Governance Reforms in the Philippines, International Journal of Water, doi: 10.1080/07900627.2015.1060196, Resources Development, 32(1), 135-152 (2016)

Sampaio, E.P., Estudio de las Prácticas Culturales, Porosidad del Suelo y Gestión Hídrica en el Combate contra la Desertificación, doi: 10.1612/inf.tecnol.4012it.08, Información Tecnológica, 20(3), 101-112 (2009)

Singh, N., Indigenous Water Management Systems: Interpreting Symbolic Dimensions in Common Property Resource Regimes, doi: 10.1080/08941920500519297, Society and Natural Resources, 19(4), 357-366 (2006)

Tahmasebi, A., Indigenous Knowledge for Water Management in Iran's Dry Land - Siraf, doi: 10.1080/00207230902722481, International Journal of Environmental Studies, 66(3), 37-41 (2009)

Tomaselli, K.G., Who owns what? Indigenous Knowledge and Struggles over Representation, doi: 10.1080/02560046.2014.929220, Critical Arts: South-North Cultural and Media Studies, 28(4), 37-41 (2014)

Uprety, Y., H. Asselin y otros tres autores, Contribution of Traditional Knowledge to Ecological Restoration : Practices and Applications, doi: 10.2980/19-3-3530, BioOne, 19(3), 225-237 (2012)

Wateau, F., Water, Societies and Sustainability: A few Anthropological Examples of Non-Market Water, Values, doi: 10.1016/j.polsoc.2011.10.004, Policy and Society, 30(4), 257-265 (2011)

Williams, T. y P. Hardison, Culture, Law, Risk and Governance: Contexts of Traditional Knowledge in Climate Change Adaptation, doi: 10.1007/s10584-013-0850-0, Climatic Change, 120, 531-544 (2013) 